\title{
Neutrophil Transmigration: Emergence of an Adhesive Cascade within Venular Walls
}

\author{
Mathieu-Benoit Voisin Sussan Nourshargh \\ William Harvey Research Institute, Barts and The London School of Medicine and Dentistry, Queen Mary University \\ of London, London, UK
}

\section{Key Words}

Abluminal crawling · Adhesion molecules · Basement membrane · Endothelial cells · Low expression regions . Neutrophils · Pericytes · Trans-/paracellular migration . Transmigration - Venular basement membrane

\section{Abstract}

Recruitment of neutrophils from the blood circulation to sites of infection or injury is a key innate immune response against invading pathogens and tissue injury. However, if inappropriately triggered, excessive and/or prolonged, this host defence response can also lead to severe pathological disorders. The migration of all leucocytes out of the vasculature is classically described by the leucocyte adhesion cascade that depicts a well-characterised sequence of cellular and molecular events within the vascular lumen. Recent findings have now illustrated that beyond the vascular lumen, the breaching of the venular wall can also involve an analogous cascade of adhesive events. For neutrophils this involves a tightly regulated and sequential series of responses within venular walls, initiating with adhesive steps that guide neutrophils through endothelial cells lining the venular wall, followed by responses that mediate and regulate their migration through the pericyte sheath and the venular basement membrane. The present review aims to provide a brief summary of novel additions to the classical adhesion cascade within the vascular lumen and then to discuss the emergence of a second adhesion cascade for neutrophils within venular walls, the latter illustrating the intricacies and complexities of neutrophil transmigration.

Copyright $\odot 2013$ S. Karger AG, Basel

\section{Introduction}

Blood neutrophils are relatively short-lived cells $(<6$ days) and are the most abundant leucocyte population in human blood (40-60\%). Nearly $10^{11}$ of these cells are generated in the bone marrow and released into the blood circulation every day. In contrast to other components of the innate immune system, such as macrophages, dendritic cells and mast cells that reside in extravascular tissues, neutrophils have to undergo an elaborate and complex response of migrating out of the vascular lumen, most notably in post-capillary venules, in order to exert their host-defence actions in tissues. In this context, neutrophils are the first leucocytes to arrive at sites of infection or tissue injury where they exhibit numerous effector functions such as killing and phagocytosis of invading

\section{KARGER}

E-Mail karger@karger.com

www.karger.com/jin (c) 2013 S. Karger AG, Basel

$1662-811 \mathrm{X} / 13 / 0054-0336 \$ 38.00 / 0$
Prof. Sussan Nourshargh

Centre for Microvascular Research, William Harvey Research Institute Barts and The London School of Medicine and Dentistry

Queen Mary University of London, Charterhouse Square, London EC1M 6BQ (UK)

E-Mail s.nourshargh@ @mul.ac.uk 
pathogens. Furthermore, neutrophils have been shown to generate pro-inflammatory cytokines and chemokines for the recruitment of other leucocytes and contribute to the development of Th1 inflammatory responses via the generation of IFN $\gamma$. Hence, in addition to an immediate role, neutrophils have also been implicated in numerous adaptive immune responses, suggesting a broader role for these cells than classically considered. The ability of neutrophils to generate a wide array of cytotoxic molecules (e.g. proteases and reactive oxygen metabolites) means that excessive recruitment of activated neutrophils can also cause damage to the host and contribute to the development of inflammatory disorders. As a result, neutrophils have been implicated in the pathogenesis of both acute (e.g. myocardial infarction and stroke) and chronic (e.g. rheumatoid arthritis, atherosclerosis, vasculitis and asthma) inflammatory conditions [1].

Since the first applications of microscopy to visualise the migration of leucocytes out of blood vessels more than 150 years ago, the mode and mechanisms of this response have fascinated inflammation and immunology researchers. In the modern era of science, advancements in highresolution in vivo imaging techniques, as applied to the study of genetically modified animals, have considerably enhanced our understanding of how leucocytes migrate into tissues. It is now well known that neutrophil movement out of blood vessels involves a complex cascade of cellular and molecular responses (fig. 1) that initiates with selectin-mediated tethering and rolling of leucocytes along the luminal side of the endothelium adjacent to the site of infection/injury followed by chemokine-induced integrinmediated firm adhesion and luminal crawling of leucocytes [2]. Subsequently, neutrophils are required to migrate through venular walls, a response prerequisite to their emigration into the extravascular space and movement towards the core of the inflammatory insult [3]. Whilst the total duration of luminal neutrophil-vessel wall interactions can be very rapid (e.g. about 5-10 min or less), neutrophil migration through venular walls can take significantly longer (e.g. about $20-45 \mathrm{~min}$ or more) with much of this delay being accounted for by post-endothelial cell (EC) events. This stage of leucocyte trafficking has largely been neglected due to the difficulties associated with the isolation of pure primary pericytes and hence the in vitro reconstitution of a complete venular wall construct incorporating ECs, pericytes and a physiologically relevant vascular basement membrane (BM) structure generated by both of these cells. Recent studies have however begun to shed light on post-EC neutrophil responses within venular walls, addressing the mode, dynamics and mechanisms of

Neutrophil Transmigration: A Cascade of Events within Venular Walls neutrophil interactions with pericytes and identifying specific exit sites within the venular BM that are targeted by transmigrating neutrophils. The present article provides a brief overview of luminal neutrophil-venular wall interactions, highlighting some novel concepts, followed by a more detailed review of key cellular and molecular events during neutrophil migration through venular walls.

\section{From Capture to Sites of Transendothelial Cell Migration}

Neutrophil recruitment at sites of infection or sterile injury is initiated by the release of pathogen-associated molecular patterns from invading micro-organisms or damage-associated molecular patterns derived from damaged and/or dead cells, respectively [4]. These danger molecular signals are recognised locally by tissue-resident innate immune cells such as resident macrophages, dendritic cells, fibroblasts and ECs that express surface and/ or intracellular pattern recognition receptors. These include Toll-like receptors, C-type lectin receptors, NODlike receptors and RIG-I-like receptors [5], activation of which can lead to the generation and secretion of cytokines (e.g. IL-6, IL-1 $\beta$ and TNF), chemokines (e.g. CXCL1/2) and other neutrophil chemo-attractants (e.g. $\mathrm{LTB}_{4}$ and platelet-activating factor), promoting leucocyte migration and the inflammatory response [6].

Cytokines in particular have a profound effect on the physiology of ECs, inducing the expression of adhesion molecules and the presentation of chemokines on the luminal surface of ECs. Among the first adhesion molecules expressed on stimulated ECs are the selectins, P- and Eselectin, that can be expressed within minutes or hours, respectively. These molecules that are largely localised to the luminal surface of ECs, bind to heavily $o$-glycosylated carbohydrate ligands, such as P-selectin glycoprotein ligand (PSGL)-1, CD44 and E-selectin ligand-1 present on the surface of free-flowing neutrophils, supporting neutrophil tethering and rolling [7]. A recent addition to our understanding of how neutrophils can maintain a rolling contact with venular walls at high shear stress is the identification of novel cellular structures termed 'slings' [8]. In an elegant study, Sundd et al. [8] demonstrated that upon high shear stress, to aid their slowing down, neutrophils extend long membrane extensions at the front of the cell. These structures (slings) wrap around neutrophils like a lasso and through discontinuous expression of PSGL-1 provide intermittent sticky patches along the sling that serve to provide a unique adhesive substrate for rolling 


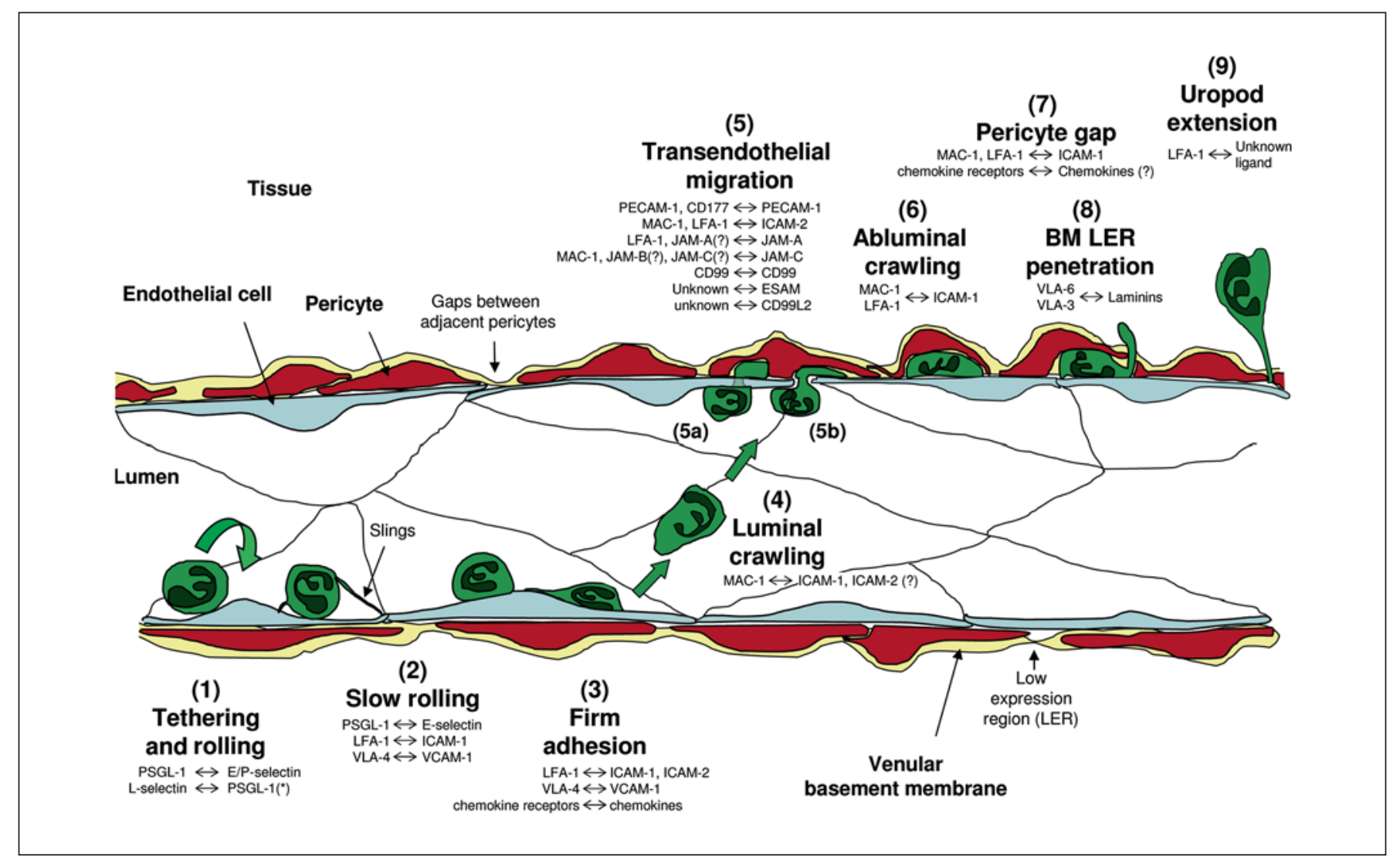

Fig. 1. The neutrophil adhesion cascade. The diagram depicts a simplified illustration of the neutrophil adhesion cascade that mediates migration of neutrophils from the vascular lumen to the extravascular tissue. The distinct steps are indicated by numbers (1-9) and the key associated molecular pathways are listed with the molecules named on the left-hand side being neutrophil-expressed and on the right-hand side being expressed by the vessel wall (i.e. ECs, pericytes or venular BM). * The ligand of neutrophil-expressed L-selectin is neutrophil-derived PSGL-1 (i.e. neutrophil rolling on adherent neutrophils or EC-bound neutrophil particles). The initial step is (1) the capture and rolling of neutrophils on activated ECs followed by (2) slow rolling (largely mediated via selectin signalling) and (3) firm adhesion to ECs through strong adhesive contacts between leucocytes and ECs mediated through activation of neutrophil integrins by chemo-attractants such as chemokines. These interactions induce intracellular signals within the neutrophils that are responsible for cytoskeletal rearrange-

neutrophils. Slings appear to occur when a neutrophil membrane tether, in the form of an EC-bound anchor left at the back of the rolling neutrophil, swings round to the front of the cell and reattaches to ECs. The slings accumulate over time and as such strengthen and stabilise the slowing down of neutrophils on the endothelium. Interestingly, these structures are also enriched in the neutro- ments and polarisation of the cell. This leads to (4) luminal motility (or luminal crawling) along the endothelium until they reach their site of (5) TEM. Neutrophils can cross the endothelium by either (5a) transcellular or (5b) transjunctional routes (the latter being more prevalent). This step involves complex interactions involving numerous EC junctional molecules. Once through the endothelium, neutrophils exhibit (6) abluminal crawling along pericyte processes. At this step, neutrophils will also be interacting with the venular BM, most probably via integrin-mediated mechanisms. Neutrophils eventually breach the pericyte layer (7) by migrating through gaps between adjacent cells and (8) regions of low matrix protein deposition (LERs) in the BM. To finally migrate into the interstitial tissue, neutrophils need to detach from the vessel wall (9), a response that is illustrated through the formation of an elongated uropod that must be detached before neutrophils can initiate their movement towards the inflammatory foci.

phil integrin lymphocyte function-associated antigen (LFA)-1 $\left(\alpha_{\mathrm{L}} \beta_{2}\right)$, which can interact with its ligand ICAM2 expressed largely on the neutrophil cell surface in trans. Stabilised rolling can develop into slow rolling via activation of LFA-1 through the binding of E-selectin to neutrophil PSGL-1 in an Src kinase spleen tyrosine kinase (Syk)mediated manner [9]. This allows LFA-1 to interact with 
its EC ligand ICAM-1, an interaction that supports slow rolling of neutrophils along the endothelium [10]. By this stage, neutrophil-EC contact is greatly enhanced and neutrophils are better enabled to screen the surface of the endothelium for activating molecules. This allows neutrophils to detect soluble chemo-attractants, such as chemokines generated at sites of tissue damage or infection, that may be immobilised on the surface of the endothelium via glycosaminoglycans [11]. Of interest, it has been proposed that newly generated chemokines may be expressed on the luminal side of venular ECs through transcytosis from the abluminal side via the Duffy antigen receptor for chemokines [12]. Detection of chemokines and other lipid (e.g. $\mathrm{LTB}_{4}$ and platelet-activating factor) or protein (e.g. C5a and LL37) chemo-attractants induce intracellular signals via the activation of G-protein-coupled receptors on neutrophils. These signals lead to full arrest of neutrophils on the endothelium by cytoskeletal rearrangement and activation of integrins such as LFA-1. The cascade of molecular events that regulate the transition from neutrophil rolling to firm arrest has been extensively studied and is well documented in the literature [13]. In addition to the critical role of $\beta_{2}$ integrins, the $\beta_{1}$ integrin very-late antigen (VLA)-4 $\left(\alpha_{4} \beta_{1}\right)$ has also been implicated in neutrophil firm attachment. Whilst VLA-4 is significantly expressed on the surface of resting murine neutrophils [14], its expression on human neutrophils is negligible but can be up-regulated following stimulation. As a result, it has been suggested that under certain conditions, neutrophils may adhere independently of $\beta_{2}$ integrins and employ VLA-4 for firm attachment to ECs [15].

One of the major effects of firm adhesion is a rapid change in neutrophil morphology characterised by rearrangement of the actin cytoskeleton, flattening and polarisation of the cell with an invasive lamellipodium at the leading edge and a uropod at the rear. This polarisation is the consequence of both haptotactic gradients of chemoattractants activating their cognate receptors (e.g. chemokines activating G-protein-coupled receptors) as well as following the interaction of neutrophil and EC adhesion molecules. Collectively, these events induce distinct signalling pathways that lead to F-actin polymerisation at the front and actin-myosin contraction and retraction of the plasma membrane at the back of the neutrophil. This supports neutrophil crawling along the luminal surface of the endothelium, and significant progress has been made in elucidating the details of associated intracellular pathways [16]. Neutrophil crawling is now well accepted as a distinct and major cellular component of the leucocyte adhesion cascade. Indeed, advances in real-time imaging of neutro-

Neutrophil Transmigration: A Cascade of Events within Venular Walls
phil-EC interactions in vivo have shown that after firm adhesion, neutrophils can exhibit significant luminal crawling in a MAC-1 $\left(\alpha_{\mathrm{M}} \beta_{2}\right) / \mathrm{ICAM}-1$-dependent manner, covering distances of up to $60 \mu \mathrm{m}$ (for $\sim 6-10 \mathrm{~min}$ ), before finding their preferred site of transendothelial cell migration (TEM) [17]. The transition between LFA-1-dependent firm arrest and MAC-1-dependent crawling is not fully understood, but there is evidence for the involvement of LFA-1-mediated outside-in signalling and subsequent intracellular activation of Vav-1 protein [18], a guanine exchange factor previously shown to be involved in MAC-1 activation [19]. Interestingly, Phillipson et al. [18] also found that neutrophil luminal crawling can occur independently of blood flow direction. Indeed, neutrophil luminal motility largely occurs perpendicularly to the direction of the blood stream, a response that may support a more efficient integrin-mediated neutrophil attachment under conditions of flow. This perpendicular crawling was absent in Vav-1-/- neutrophils suggesting that shear stress-mechanosensing receptors may contribute to the observed Vav1- and MAC-1-dependent crawling. It is hypothesized that once the neutrophils have reached an EC junction, haptotactic gradients of surface-bound chemokines/chemo-attractants and adhesion molecules expressed at the intercellular junctions of ECs take over the mechanotactic signals and collectively guide neutrophils into and through EC junctions, as detailed below.

\section{Neutrophil Transmigration: The Emergence of a Distinct Adhesive Cascade}

To exit the vascular lumen, neutrophils need to breach the venular wall, a step in leucocyte trafficking that can take $>20-45 \mathrm{~min}$. The dynamics of this response is the first indication that neutrophil migration through venular walls involves multiple interactions with different components of this structure, namely the endothelium, pericytes and their associated BM (fig. 2). Analogous to the luminal adhesive interactions detailed above, emerging data demonstrate that neutrophil transmigration similarly occurs via a series of adhesive interactions, as described below.

\section{Neutrophil TEM}

The first step in neutrophil transmigration is breaching of the EC barrier. To achieve this, neutrophils can penetrate ECs via two routes: through junctions between 


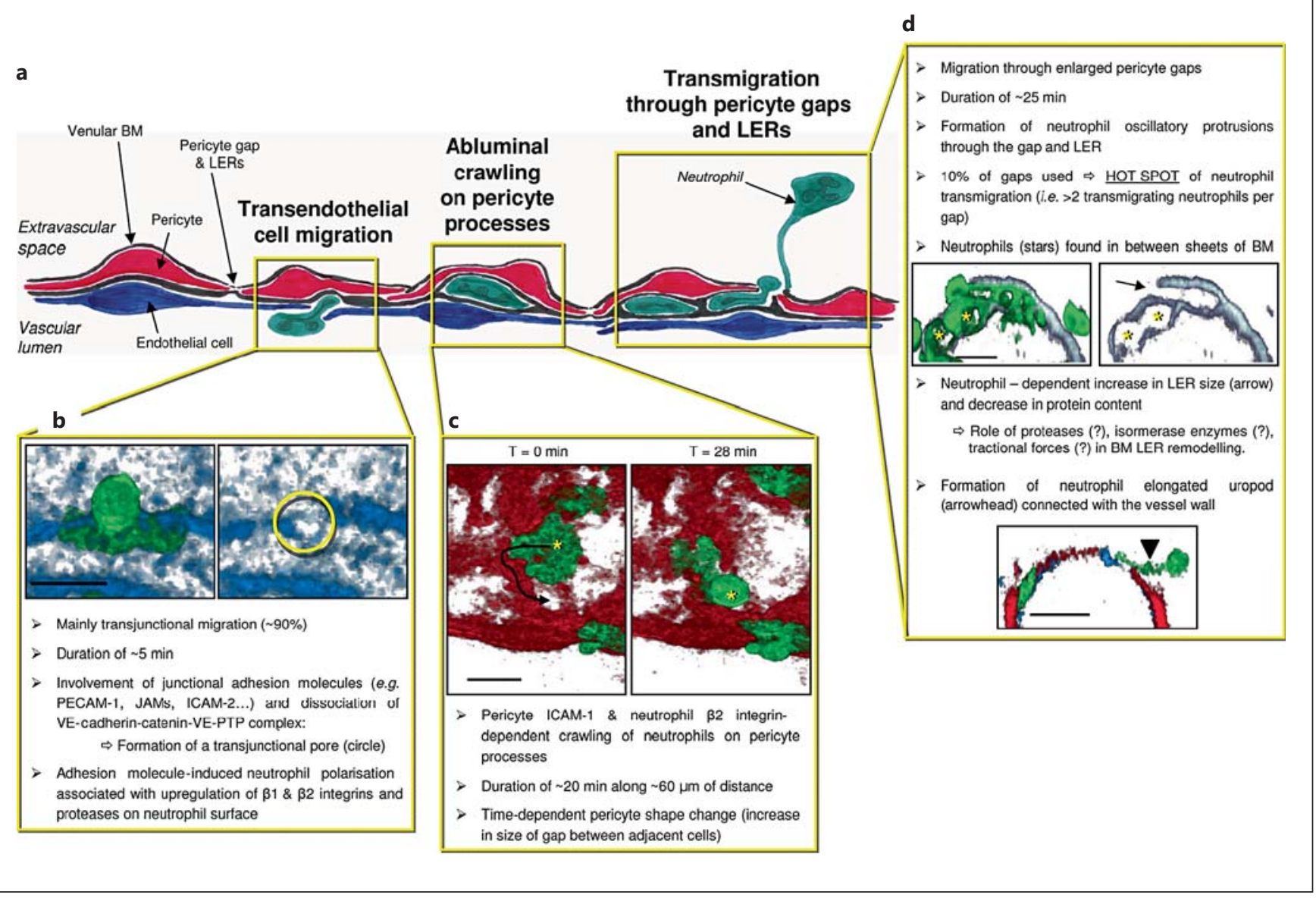

Fig. 2. Neutrophil transmigration through venular walls. a Simplified illustration of the sequential migration of neutrophils through different cells/structures of the venular wall: ECs, pericytes and the vascular BM within which pericytes are embedded. b To breach venular walls, neutrophils first need to migrate through ECs. This is a fast process $(\sim 5 \mathrm{~min})$ that can involve multiple adhesive interactions. The process involves the formation of a pore within EC junctions (transjunctional route, the principal route: neutrophil TEM) or the body of ECs (transcellular route). TEM is also associated with phenotypic changes and polarisation of neutrophils. The images are $3 \mathrm{D}$ reconstructed images of TNF-stimulated cremasteric venules from $L y s M-e G F P$ - $k i$ mice exhibiting fluorescent neutrophils (green) that also have fluorescently labelled ECs using an anti-PECAM-1 mAb (blue). Extravasation was observed by live confocal microscopy and only one time point of the TEM process is shown. In both panels, the vessel is viewed from the luminal side with (left panel) or without (right panel) the neutrophil being visible to highlight the transjunctional pore (circle). c Once through the EC barrier, neutrophils exhibit sub-endothelial crawling along pericyte processes. Images in the insert are from live confocal observations of a venule in a TNF-stimulated cremaster muscle of a genetically modified mouse exhibiting both endogenous fluores- cent neutrophils (LysM eGFP, green) and pericytes (aSMAcherry-RFP, red) and in vivo labelling of EC junctions using an anti-PECAM-1 mAb (blue). The 2 panels are $3 \mathrm{D}$ reconstructed images viewed for the extravascular space showing the distance of neutrophil migration along pericyte processes (arrow). d Sub-endothelial motility enables neutrophils to seek specific permissive sites within the pericyte layer (i.e. gaps between adjacent cells) and the venular BM (LERs) as exit points within the vessel wall. These events commonly involve the formation of multiple protrusions directed toward the extravascular space, and the remodelling of LERs. The top images show cross sections of a TNF-inflamed venule immunostained for BM (laminin, grey) and neutrophils (MRP14, green) as observed by confocal microscopy. Only a small percentage of pericyte gaps and LERs are used by migrating neutrophils, resulting in the existence of 'hot spots' that support transmigration of multiple neutrophils. As neutrophils exit the vessel, an elongated uropod is often noted attached to the venular wall (bottom image, cross section showing neutrophils in green, pericytes in red and EC-PECAM-1 in blue), a cellular structure that requires retraction before neutrophils can fully enter the interstitial space. Bar $=10 \mu \mathrm{m}$. 
adjacent ECs (i.e. transjunctional or paracellular migration) or through the body of the endothelium (i.e. transcellular migration). Both modes of neutrophil TEM have been extensively studied and reviewed in the literature [3, $20,21]$. Overall, the current evidence from both in vitro and in vivo studies indicates that junctional migration is the most prominent mode of breaching the endothelium, i.e. $\sim 70-90 \%$. This was clearly illustrated in the murine cremaster muscle microcirculation, as observed and quantified using an advanced confocal intravital microscopy platform, where paracellular TEM was found to account for $\sim 90 \%$ of all observed neutrophil TEM events induced by multiple inflammatory stimuli [22]. Compelling evidence for the importance of EC junctions in neutrophil TEM was also obtained in an innovative series of studies by Schulte et al. [23] using a novel genetic approach. Briefly, through genetic insertion of a vascular endothelial (VE)-cadherin- $\alpha$-catenin fusion complex (replacing endogenous VE cadherin), a novel mouse strain was generated in which the blood vessels were resistant to permeability-inducing factors [23]. The use of this approach for stabilisation of EC junctions also suppressed leucocyte (neutrophil and lymphocyte) infiltration in several models of inflammation, providing an elegant means of obtaining supportive data for a key role for EC junctions in leucocyte trafficking $[16,20]$. VE cadherin, which under basal conditions associates in a homophilic manner with VE cadherin expressed on adjacent ECs, also associates in cis with the transmembrane VE protein tyrosine phosphatase and $\alpha$-catenin (via $\beta$-catenin). Using yet another novel genetic approach, Vestweber [20] and others [24] have also acquired in vivo evidence to show that the dissociation of VE cadherin and protein tyrosine phosphatase is necessary for opening of EC junctions during neutrophil trafficking. It is thus proposed that the VE-cadherin-catenin-VE-protein tyrosine phosphatase complex acts as a key gate keeper of endothelial junctions, and its destabilisation during inflammation induced by neutrophil adhesion and clustering of ICAM-1 and VCAM-1 on the luminal surface of the endothelium $[24,25]$ is an essential step in the migration of neutrophils through this junctional barrier. Whilst the above recent studies provide conclusive evidence for the importance of junctional migration, it remains possible that the frequency of transcellular TEM is governed by the vascular bed and/or the nature and phase of the inflammatory reaction. Of interest, it has been suggested that the route of neutrophil TEM through dermal microvascular ECs is dependent on the levels of endothelial caveolin-1, the protein responsible for the induction of the membrane

Neutrophil Transmigration: A Cascade of Events within Venular Walls domains (caveolae). Briefly, caveolin-1 expression was found to favour transcellular TEM while down-regulation of caveolin-1 promoted neutrophil paracellular TEM [26], suggesting that EC expression of caveolin-1 may be a key factor in determining the route of neutrophil TEM.

Transendothelial and transjunctional (paracellular) migration of neutrophils exhibits common features such as the use of a number of adhesion molecules that are enriched at EC junctions but also detected within EC intracellular structures [21]. These include platelet EC adhesion molecule-1 (PECAM-1), members of the junctional adhesion molecule (JAM) family, ICAM-2, CD99, CD99L2 and EC-selective adhesion molecules. These molecules contribute to maintaining the integrity of junctions between adjacent ECs, and largely through interactions with their counter receptors on the surface of neutrophils play an active role in mediating leucocyte transmigration. A number of these molecules appear to support different stages of neutrophil transmigration and also to mediate this response in a stimulus-specific manner [3, 21]. Furthermore, there is increasing evidence for the regulation of expression of EC adhesion molecules by inflammatory stimuli both in a temporal and spatial manner. For example, the binding of neutrophils to and clustering of ICAM1 on the luminal surface of ECs can induce the activation of intracellular signalling pathways (via Scr kinase and eNOS pathways) in ECs leading to an increase in PECAM-1 phosphorylation and up-regulation of its expression [27]. This EC up-regulation of surface PECAM-1 is linked with the mobilisation of the molecule from intracellular structures close to EC junctions termed the lateral border recycling compartment [28]. Other junctional molecules that are regulated during inflammation include JAM-A and JAM-C $[29,30]$. These adhesion molecules have also been detected in intracellular structures, with JAM-A being found in the lateral border recycling compartment in vitro [31] and JAM-C being localised to small cytoplasmic vesicles ( $\sim 60 \mathrm{~nm}$ in diameter) in vivo [30]. At EC junctions, these molecules are within tight junctions were they form homophilic (JAM-A-JAM-A and JAM-CJAM-C) or heterophilic (JAM-C-JAM-B) interactions, and the disruption of these interactions can lead to the redistribution of the molecules to the apical surface of ECs. Such a response could potentially impact the stabilisation, integrity or barrier function of EC junctions, for which there is some evidence [32]. Relocalisation of JAM-A and JAM-C to the apical surface of ECs could also play a role in promoting the luminal adhesion of neutrophils and in providing a haptotactic gradient of these adhesion molecules from the apical side of ECs to the junctions. In sup- 
port of this, blockade of JAM-A suppresses neutrophil penetration of EC junctions, possibly via blocking the interaction of luminally expressed EC JAM-A with its neutrophil ligand LFA-1 [33]. JAM-A expressed on neutrophils also contributes to leucocyte trafficking in that it mediates polarisation and migration of neutrophils both in vitro in response to chemotactic gradients and in vivo during interstitial migration in inflamed tissues [34]. Mechanistically, JAM-A-null neutrophils exhibit impaired internalisation and recycling of $\beta_{1}$ integrins and fail to activate the small GTPase RAP-1 involved in cytoskeletal rearrangement and cell polarisation. Whether a similar mechanism contributes to JAM-A-dependent neutrophil polarisation and migration through EC junctions in vivo is at present unknown. Interestingly, EC JAM-C has recently been demonstrated to play a critical role in polarised luminal to abluminal neutrophil TEM. Through the application of confocal intravital microscopy to the analysis of neutrophil-EC interactions in inflamed mouse cremasteric venules, we have reported on the occurrence of a number of novel modes of neutrophil TEM [22]. Specifically, we noted that inflammatory conditions that resulted in reduced expression of EC JAM-C (e.g. ischaemia-reperfusion injury), or following genetic deletion or pharmacological blockade of EC JAM-C, neutrophils exhibited disrupted modes of junctional motility characterised by abluminal to luminal movement [22]. The transparency of zebrafish embryos also offers an excellent model for tracking of myeloid cells, and within this system there is also evidence for unexpected neutrophil motility, i.e. migration of neutrophils from inflamed tissues back into the vascular lumen [35]. It should be noted, however, that the zebrafish immune system is very different from the mammalian system, and hence the associated mechanisms and functional implications of specific EC adhesion molecules in neutrophil migration are unknown. Interestingly, in vitro studies have shown that reverse TEM neutrophils show a distinct phenotype (e.g. are ICAM-1 $1^{\text {high }}$ and show an enhanced pro-inflammatory state) [36] and in mice subjected to hind-limb ischaemia-reperfusion injury, the presence of ICAM-1 ${ }^{\text {high }}$ neutrophils in the pulmonary vasculature correlated with lung inflammation [22]. These findings have led us to suggest that abluminal to luminal migration of neutrophils, as caused by loss of EC junctional JAM-C, leads to return of an activated subset of neutrophils back into the circulation that can in turn contribute to spreading a localised inflammatory response to secondary organs [22]. This novel paradigm for dissemination of systemic inflammation is currently under investigation in the context of other inflammatory conditions.

\section{Pericytes: An Additional Cellular Player in the Regulation of Neutrophil Transmigration}

Once through the endothelium, neutrophils encounter the second cellular component of venular walls, pericytes, mural cells that are typically embedded within the vascular BM. Pericytes are elongated cells that are ubiquitously expressed in microvessels, but their expression profile and phenotype can vary considerably in different types of microvessels, tissues and species [37]. Indeed, the lack of a single unifying pericyte marker has significantly slowed down investigations into the functions of these cells in vitro and in vivo. Nonetheless, there is considerable evidence to suggest a role for these contractile cells in cardiovascular homeostasis and disease [37]. In the context of inflammation, numerous in vitro studies have shown that like ECs, pericytes can express key adhesion molecules, most notably under inflammatory conditions (table 1). For example, VCAM-1 was found on pericytes in human rheumatoid synovial tissue and human brain of patients with inflammatory neuropathology, and ICAM1 was detected in skin samples from patients with bullous pemphigoid $[38,39]$. Pericytes isolated from the bloodbrain barrier up-regulated both ICAM-1 and VCAM-1 in response to TNF stimulation, and supported T-cell adhesion in a VLA-4-VCAM-1-dependent manner [40]. More recently, Maier and Pober [41] reported that human placental pericytes did not express VCAM-1 or E-selectin, but did express ICAM-1, which could be further induced following stimulation of the cells by the cytokines IFN $\gamma$ and TNF.

Very few studies have addressed the role of pericytes in neutrophil transmigration. Through analysis of whole mounted inflamed murine cremaster muscles, we observed that neutrophils breach venular walls by migrating through gaps between adjacent pericytes, sites that also expressed a lower deposition of BM constituents (see following section) $[42,43]$. More recently, we have provided the first direct evidence for neutrophil-pericyte interactions at sites of inflammation in real time in vivo, demonstrating that this interaction plays a key role in facilitating neutrophil transmigration through venular walls [44]. Specifically, through the use of genetically modified mice exhibiting RFPcherry pericytes and smooth muscle cells (under the control of the aSMA promoter) and enhanced green fluorescent protein (eGFP) myeloid cells (LysM-eGFP-ki mice), neutrophil-pericyte interactions in post-capillary venules were observed and analysed in the mouse cremaster muscle microcirculation in real time in $3 \mathrm{D}$ by confocal intravital microscopy 
Table 1. Expression and function of adhesion molecules on pericytes

\begin{tabular}{|c|c|c|c|c|}
\hline $\begin{array}{l}\text { In vitro } \\
\text { Bovine retinal } \\
\text { capillary pericytes }\end{array}$ & N/D & $\begin{array}{l}\text { glycosaminoglycan } \\
\text { (heparin sulfate, } \\
\text { hyaluronic acid) }\end{array}$ & $\mathrm{N} / \mathrm{D}$ & $\begin{array}{l}\text { Stramm et al. [54], } \\
1987\end{array}$ \\
\hline $\begin{array}{l}\text { Mouse brain } \\
\text { microvascular pericytes }\end{array}$ & $\mathrm{IFN} \gamma$ & N/A & $\begin{array}{l}\text { antigen-specific activation } \\
\text { of Th1 cells }\end{array}$ & $\begin{array}{l}\text { Fabry et al. [56], } \\
1993\end{array}$ \\
\hline $\begin{array}{l}\text { Rat brain microvessel } \\
\text { pericytes }\end{array}$ & TNF, IFN $\gamma$ & ICAM-1, VCAM-1 & $\begin{array}{l}\text { antigen-specific activation } \\
\text { of splenocytes }\end{array}$ & $\begin{array}{l}\text { Balabanov et al. [57], } \\
1999\end{array}$ \\
\hline $\begin{array}{l}\text { Human placental } \\
\text { pericytes }\end{array}$ & TNF & ICAM-1 & anergy of T cells & $\begin{array}{l}\text { Maier and Pober [41], } \\
2011\end{array}$ \\
\hline $\begin{array}{l}\text { In vivo } \\
\text { Human MS brain } \\
\text { biopsy sections }\end{array}$ & N/A & VCAM-1 & $\mathrm{N} / \mathrm{D}$ & $\begin{array}{l}\text { Verbeek et al. [40], } \\
1995\end{array}$ \\
\hline $\begin{array}{l}\text { Human RA synovium } \\
\text { biopsy sections }\end{array}$ & N/A & VCAM-1 & N/D & $\begin{array}{l}\text { Kriegsmann et al. [39], } \\
1995\end{array}$ \\
\hline $\begin{array}{l}\text { Human bullous pemphigoid } \\
\text { dermal biopsy sections }\end{array}$ & N/A & $\begin{array}{l}\text { ICAM-1, VCAM-1, } \\
\text { E-selectin }\end{array}$ & $\mathrm{N} / \mathrm{D}$ & $\begin{array}{l}\text { Dahlman-Ghozlan et al. } \\
\text { [59], } 2004\end{array}$ \\
\hline
\end{tabular}

$\mathrm{N} / \mathrm{A}=$ Not applicable; N/D = not done; $\mathrm{MS}=$ multiple sclerosis; $\mathrm{RA}=$ rheumatoid arthritis.

[44]. In TNF-stimulated tissues, we observed that after TEM neutrophils crawled along pericyte processes for $\sim 20 \mathrm{~min}$ at a speed of $\sim 3 \mu \mathrm{m} / \mathrm{min}$, thus covering a total distance of $\sim 60 \mu \mathrm{m}$ in a meandering manner (this is equivalent to $\sim 20 \mu \mathrm{m}$ total displacement from the site of TEM and to the point of breaching the pericyte sheath). In agreement with the findings of human placental pericytes in vitro, cremasteric venular pericytes expressed low levels of ICAM-1 that was up-regulated in TNFstimulated tissues. Furthermore, neutrophil abluminal crawling was mediated by ICAM- 1 and also the $\beta_{2}$ integrins Mac-1 and to a lower extent LFA-1. Although the majority of the pericyte-EC interface is separated by the $\mathrm{BM}$, the two cells are in close vicinity of each other and indeed do make contact at discrete points [37]. Because of this, at present, it is unclear what the relative contributions of EC and pericyte ICAM-1 are in the noted ICAM1 -mediated sub-EC crawling. Having said that, our findings showed that the path of neutrophil crawling within post-capillary venular walls was almost totally governed by the shape and anatomy of pericytes and their processes, strongly suggesting that pericytes provide adhesive tracks for abluminal neutrophil crawling. The potential role of other adhesion pathways, e.g. involving ICAM-2 and VCAM-1, in this process requires investigations, but our current data indicate that ICAM-2 is not expressed to a notable level on mouse cremaster muscle venular pericytes and that VCAM-1 is not induced within the acute reactions (4-hour TNF stimulation) we have studied. Following abluminal motility, neutrophils migrate 
through specific gaps between adjacent pericytes, and no transcellular migration through the body of pericytes was noted. This is in contrast to a previous electron-microscopic study that reported on significant neutrophil transcellular migration through pericytes in a model of guinea pig dermal inflammation [45], supporting again the concept that the route and mode of neutrophil transmigration can vary in different vasculature beds and/or inflammatory conditions. Interestingly, real-time analysis of inflamed tissues indicated that $<10 \%$ of pericyte gaps were used by transmigrating neutrophils [44]. The factors that determine which pericyte gaps are employed by emigrating neutrophils is at present unclear and may be ascertained by physically (e.g. size) and/or biochemically (e.g. existence of guidance molecules) desirable regions and indeed multiple determinants may be involved. For instance, we have found that in cytokine (TNF and IL-1 $\beta$ )-stimulated tissues pericyte gaps are enlarged in a time-dependent manner and that neutrophils avoid both the very small $\left(<8-\mu \mathrm{m}^{2}\right.$ area $)$ and the very large $\left(>50-\mu \mathrm{m}^{2}\right.$ area) gaps between adjacent pericytes [44]. Furthermore, we have identified a small subset of pericytes enriched with ICAM-1 and the keratinocyte-derived chemokine at their borders which we hypothesise may guide neutrophils to exit sites within the pericyte sheath, analogous to ICAM-1-enriched EC junctions previously associated with preferential sites of neutrophil TEM [46]. The functional implications of pericyte gap enlargement during inflammation are at present unclear, but one can speculate multiple roles. For example, this phenomenon could (i) facilitate leucocyte transmigration by reducing the barrier function of the pericyte layer; (ii) contribute to an increase in diffusion of chemotactic proteins and lipids from the inflamed tissue into the vasculature to attract neutrophils and other leucocytes, and (iii) affect the integrity and hence barrier function of the vascular BM. Interestingly, within the acute cytokine-driven models we employed, we noted the existence of clear 'hot spots' in the pericyte layer in that $\sim 70 \%$ of the gaps used by neutrophils supported more than 2 transmigration events [44]. In addition, neutrophils that followed other neutrophils to exit sites showed reduced meandering and shorter abluminal crawling distances, resulting in an increased speed in exiting the pericyte layer. These observations suggest that the leading neutrophil might delineate the route taken by other leucocytes and thus facilitate the migration of the followers. The mechanism by which this occurs is at present not fully understood but may involve the release of chemo-attractants by the leading neutrophils $[16,47]$ and/or protease cleavage of BM components into peptide fragments with chemotactic activities [48], a response that could also simply pave the way within the vascular BM.

\section{Neutrophil Breaching of the Venular BM and Beyond}

The venular BM is composed of a thin extracellular matrix protein structure containing tightly organised networks of laminins (mainly isoforms -411 and -511) and collagen type IV that are interconnected by molecular interactions involving nidogens and perlecan glycoproteins [49]. Generated by both ECs and pericytes, this structure encapsulates pericytes and contributes to the barrier function of venular walls both for macromolecules and for emigrating leucocytes. Investigations into mechanisms through which neutrophils breach this component of venular walls is fraught due to difficulties in creating physiologically relevant in vitro models of venular BMs (e.g. generated by both ECs and pericytes) and in fact rendered confusing due to the frequent employment of inadequate models such as Matrigel. As a result, more convincing data have stemmed from the use of in vivo models. For example, blocking experiments and/or the use of genetically modified mice have indicated roles for the $\beta_{1}$ integrin laminin receptors VLA- $6\left(\alpha_{6} \beta_{1}\right)$ and VLA$3\left(\alpha_{3} \beta_{1}\right)$ in neutrophil migration through the venular BM $[3,50]$. A significant addition to this aspect of leucocyte trafficking was our observation that the BM of post-capillary venules is a heterogeneous structure, expressing key components such as laminins and collagen IV in a nonuniform manner $[42,43]$. Structurally, this manifests into the existence of regions in the BM with low deposition of matrix protein, i.e. sites that we have termed low expression regions (LERs). The localisation and size of these regions is completely in line with gaps between adjacent pericytes, suggesting that pericytes play a key role in the generation of BM components. Importantly, in a multitude of inflammatory reactions (as induced by chemotactic molecules, cytokines and pathological insults) and tissues, LERs and their associated pericyte gaps were used by transmigrating neutrophils as exit points and were transiently remodelled (i.e. enlarged) during this process [42]. Interestingly, neutrophils appear to seek these permissive regions via extension of small protrusions [51]. Indeed live imaging of transmigrating neutrophils showed that whilst lead neutrophils can exhibit oscillatory movements associated with the formation of multiple protrusions into the venular BM before exiting the vessel wall, subsequent neutrophils can get to and breach the same 
exit point more quickly. These observations suggest that BM LERs used for penetrating the vascular BM may be modified by first-line emigrating neutrophils in a way that renders them more permissive to other leucocytes as the inflammatory response develops. The precise mechanism(s) involved in LER remodelling remains unclear, but we do know that it occurs in a strictly neutrophil-dependent manner $[43,51]$. The obvious possibilities are proteolytic cleavage of BM components [48] and/ or subtle disassembly and physical carriage of neutrophilbound BM components, most notably non-covalently expressed components such as laminin, by emigrating neutrophils [43]. Regarding the former, despite many investigations, the role of neutrophil proteases in venular BM penetration and remodelling remains a contentious issue and there exist numerous conflicting results [3, 43, 49]. Other hypotheses presented in the context of neutrophil migration through the venular BM include models related to the physical properties of the BM. For example, it has been suggested that the tightly organised network of covalently linked collagen type IV oligomers could be subjected to reversible disassembly either by tractional forces of cells penetrating the BM or by the activity of isomerase enzymes $[3,49]$. Another hypothesis suggests that BM LERs contain subunits of laminins with lower capacity for cross-linking with collagen type IV molecules (e.g. the short $\alpha_{4}$ chain of laminin -411), a phenomenon that would facilitate easier dissociation of the laminin/collagen IV networks during cell migration. Although attractive, there currently exists no direct evidence for these possibilities, and how neutrophils breach and remodel the venular BM requires further investigations.

The final step in leucocyte transmigration involves detachment of neutrophils from the venular wall. Intravital microscopists have long observed that this can involve the formation of long and thin leucocyte tails that eventually detach as the cell migrates into the interstitial tissue. A recent study has addressed the molecular basis of this uropod formation [50]. Using a knock-in mouse expressing the $\beta_{2}$ integrin subunit (CD18) fused to monomeric cherry fluorescent protein, the authors could track the distribution of endogenous CD18 during neutrophil transmigration within cremasteric venules in real time in vivo by multi-photon intravital microscopy. Briefly, it is reported that the elongated uropod occurs as a consequence of LFA-1-dependent attachment of neutrophil tails to the abluminal side of blood vessel walls [50]. The exact ligand(s) and structure (EC, pericyte and/or BM) of the vessel wall implicated in this attachment is unclear, but detachment of the uropod is speculated to be associ- ated with modulation of LFA-1 deactivation and appeared to be associated with deposition of $\mathrm{CD} 18+$ microparticles at the vessel wall. Once in the extravascular space, neutrophils are directed to the core of the inflammatory response through detection of guidance tracks created by chemokine gradients, adhesive structures and cellular scaffolds (e.g. fibroblasts) [52]. In addition, a recent study reported a role for a subset of pericytes (NG2+), largely expressed in the walls of capillaries and arterioles, as regulators of neutrophil interstitial migration. Specifically, through their expression of ICAM-1 and the chemokine MIF, NG2+ pericytes were found to provide promigratory signals to interstitial neutrophils, rendering them more efficient in moving towards inflammatory foci [53]. The interaction of interstitial neutrophils with NG2+ capillary pericytes also enhanced immune cell activation and survival of neutrophils. Collectively, these studies highlight the importance of understanding the molecular basis and consequences of neutrophil-vessel wall interactions both during breaching of venular walls and beyond.

\section{Concluding Remarks}

Despite the fact that neutrophil transmigration is a key immune response and is also intimately associated with the pathogenesis of numerous inflammatory disorders, many cellular and molecular aspects of this response remain unclear. However, sustained advancements in in vivo imaging techniques and the development of novel biomolecular tracers/tools have ensured continued progress in our understanding of the intricacies of this response. A significant step is the fact that more researchers are investigating mechanisms of this complex process beyond the endothelium, addressing neutrophil interactions with pericytes and the vascular BM, stages in the transmigration field that were largely neglected for many years. Despite this, there remain many unanswered questions regarding the mechanisms and dynamics of neutrophil interactions with different components of the venular wall and how interactions with one component prepare/prime the cell for the next phase. Most notably, a significant challenge for the future will be elucidating how the cellular responses and molecular pathways utilised under physiological inflammatory conditions are disrupted in response to pathological insults, an objective that will be critical in the design and identification of novel anti-inflammatory therapeutic strategies. 


\section{Acknowledgements}

M.-B.V. is supported by an Arthritis Research-UK Fellowship (MP/19913) and S.N. is the recipient of a Wellcome Trust Senior Investigator Award (Ref. 098291/Z/12/Z).

\section{Disclosure Statement}

The authors decline any conflicts of interest.

\section{References}

$\checkmark 1$ Nemeth T, Mocsai A: The role of neutrophils in autoimmune diseases. Immunol Lett 2012; 143:9-19.

$>2$ Ley K, Laudanna C, Cybulsky MI, Nourshargh S: Getting to the site of inflammation: the leukocyte adhesion cascade updated. Nat Rev Immunol 2007;7:678-689.

>3 Nourshargh S, Hordijk PL, Sixt M: Breaching multiple barriers: leukocyte motility through venular walls and the interstitium. Nat Rev Mol Cell Biol 2010;11:366-378.

$\checkmark 4$ Bianchi ME: DAMPs, PAMPs and alarmins: all we need to know about danger. J Leukoc Biol 2007;81:1-5.

5 Takeuchi O, Akira S: Pattern recognition receptors and inflammation. Cell 2010;140: 805-820.

-6 Sadik CD, Kim ND, Luster AD: Neutrophils cascading their way to inflammation. Trends Immunol 2011;32:452-460.

$>7$ McEver RP: Selectins: lectins that initiate cell adhesion under flow. Curr Opin Cell Biol 2002; 14:581-586.

-8 Sundd P, Gutierrez E, Koltsova EK, Kuwano Y, Fukuda S, Pospieszalska MK, et al: 'Slings' enable neutrophil rolling at high shear. Nature 2012;488:399-403.

$>9$ Zarbock A, Lowell CA, Ley K: Spleen tyrosine kinase Syk is necessary for E-selectin-induced alpha(L)beta(2) integrin-mediated rolling on intercellular adhesion molecule-1. Immunity 2007;26:773-783.

-10 Zarbock A, Ley K, McEver RP, Hidalgo A: Leukocyte ligands for endothelial selectins: specialized glycoconjugates that mediate rolling and signaling under flow. Blood 2011;118: 6743-6751.

11 Rot A: Chemokine patterning by glycosaminoglycans and interceptors. Front Biosci 2010;15:645-660.

-12 Pruenster M, Mudde L, Bombosi P, Dimitrova S, Zsak M, Middleton J, et al: The Duffy antigen receptor for chemokines transports chemokines and supports their promigratory activity. Nat Immunol 2009;10:101-108.

$>13$ Lefort CT, Ley K: Neutrophil arrest by LFA-1 activation. Front Immunol 2012;3:157.

$\checkmark 14$ Henderson RB, Lim LH, Tessier PA, Gavins FN, Mathies M, Perretti M, et al: The use of lymphocyte function-associated antigen (LFA)-1-deficient mice to determine the role of LFA-1, Mac-1, and alpha4 integrin in the inflammatory response of neutrophils. J Exp Med 2001;194:219-226.
15 Reinhardt PH, Elliott JF, Kubes P: Neutrophils can adhere via alpha4beta1-integrin under flow conditions. Blood 1997;89:38373846.

16 Williams MR, Azcutia V, Newton G, Alcaide P, Luscinskas FW: Emerging mechanisms of neutrophil recruitment across endothelium. Trends Immunol 2011;32:461-469.

17 Phillipson M, Heit B, Colarusso P, Liu L, Ballantyne CM, Kubes P: Intraluminal crawling of neutrophils to emigration sites: a molecularly distinct process from adhesion in the re cruitment cascade. J Exp Med 2006;203:25692575.

18 Phillipson M, Heit B, Parsons SA, Petri B, Mullaly SC, Colarusso P, et al: Vav1 is essential for mechanotactic crawling and migration of neutrophils out of the inflamed microvasculature. J Immunol 2009;182:6870-6878.

19 Gakidis MA, Cullere X, Olson T, Wilsbacher JL, Zhang B, Moores SL, et al: Vav GEFs are required for beta2 integrin-dependent functions of neutrophils. J Cell Biol 2004;166:273282.

20 Vestweber D: Relevance of endothelial junctions in leukocyte extravasation and vascular permeability. Ann NY Acad Sci 2012;1257: 184-192.

21 Woodfin A, Voisin MB, Nourshargh S: Recent developments and complexities in neutrophil transmigration. Curr Opin Hematol 2010;17:9-17.

22 Woodfin A, Voisin MB, Beyrau M, Colom B, Caille D, Diapouli FM, et al: The junctional adhesion molecule JAM-C regulates polarized transendothelial migration of neutrophils in vivo. Nat Immunol 2011;12:761-769.

23 Schulte D, Kuppers V, Dartsch N, Broermann A, Li H, Zarbock A, et al: Stabilizing the VEcadherin-catenin complex blocks leukocyte extravasation and vascular permeability. EMBO J 2011;30:4157-4170.

24 Broermann A, Winderlich M, Block H, Frye M, Rossaint J, Zarbock A, et al: Dissociation of VE-PTP from VE-cadherin is required for leukocyte extravasation and for VEGF-induced vascular permeability in vivo. J Exp Med 2011;208:2393-2401.

25 Allingham MJ, van Buul JD, Burridge K: ICAM-1-mediated, Src- and Pyk2-dependent vascular endothelial cadherin tyrosine phosphorylation is required for leukocyte transendothelial migration. J Immunol 2007;179: 4053-4064.
26 Marmon S, Hinchey J, Oh P, Cammer M, de Almeida CJ, Gunther L, et al: Caveolin-1 expression determines the route of neutrophil extravasation through skin microvasculature. Am J Pathol 2009;174:684-692.

27 Liu G, Place AT, Chen Z, Brovkovych VM, Vogel SM, Muller WA, et al: ICAM-1-activated Src and eNOS signaling increase endothelial cell surface PECAM-1 adhesivity and neutrophil transmigration. Blood 2012;120: 1942-1952.

28 Mamdouh Z, Chen X, Pierini LM, Maxfield FR, Muller WA: Targeted recycling of PECAM from endothelial surface-connected compartments during diapedesis. Nature 2003;421:748-753.

$>29$ Nourshargh S, Krombach F, Dejana E: The role of JAM-A and PECAM-1 in modulating leukocyte infiltration in inflamed and ischemic tissues. J Leukoc Biol 2006;80:714-718.

30 Scheiermann C, Colom B, Meda P, Patel NS, Voisin MB, Marrelli A, et al: Junctional adhesion molecule-C mediates leukocyte infiltration in response to ischemia reperfusion injury. Arterioscler Thromb Vasc Biol 2009;29: 1509-1515.

31 Mamdouh Z, Mikhailov A, Muller WA Transcellular migration of leukocytes is mediated by the endothelial lateral border recycling compartment. J Exp Med 2009;206: 2795-2808.

-32 Orlova VV, Economopoulou M, Lupu F, Santoso S, Chavakis T: Junctional adhesion molecule- $C$ regulates vascular endothelial permeability by modulating VE-cadherin-mediated cell-cell contacts. J Exp Med 2006;203:27032714.

33 Woodfin A, Voisin MB, Imhof BA, Dejana E, Engelhardt B, Nourshargh S: Endothelial cell activation leads to neutrophil transmigration as supported by the sequential roles of ICAM2, JAM-A, and PECAM-1. Blood 2009;113. 6246-6257.

34 Cera MR, Fabbri M, Molendini C, Corada M, Orsenigo F, Rehberg M, et al: JAM-A promotes neutrophil chemotaxis by controlling integrin internalization and recycling. J Cell Sci 2009;122(pt 2):268-277.

35 Yoo SK, Huttenlocher A: Spatiotemporal photolabeling of neutrophil trafficking during inflammation in live zebrafish. J Leukoc Biol 2011;89:661-667. 
-36 Buckley CD, Ross EA, McGettrick HM, Osborne CE, Haworth O, Schmutz C, et al: Identification of a phenotypically and functionally distinct population of long-lived neutrophils in a model of reverse endothelial migration. J Leukoc Biol 2006;79:303-311.

- 37 Armulik A, Genove G, Betsholtz C: Pericytes: developmental, physiological, and pathological perspectives, problems, and promises. Dev Cell 2011;21:193-215.

- 38 Dahlman-Ghozlan K, Ortonne JP, Heilborn JD, Stephansson E: Altered tissue expression pattern of cell adhesion molecules, ICAM-1, E-selectin and VCAM-1, in bullous pemphigoid during methotrexate therapy. Exp Dermatol 2004;13:65-69.

-39 Kriegsmann J, Keyszer GM, Geiler T, Brauer R, Gay RE, Gay S: Expression of vascular cell adhesion molecule-1 mRNA and protein in rheumatoid synovium demonstrated by in situ hybridization and immunohistochemistry. Lab Invest 1995;72:209-214.

40 Verbeek MM, Westphal JR, Ruiter DJ, de Waal RM: T lymphocyte adhesion to human brain pericytes is mediated via very late antigen-4/vascular cell adhesion molecule-1 interactions. J Immunol 1995; 154:5876-5884.

-41 Maier CL, Pober JS: Human placental pericytes poorly stimulate and actively regulate allogeneic CD4 T cell responses. Arterioscler Thromb Vasc Biol 2011;31:183-189.

42 Voisin MB, Probstl D, Nourshargh S: Venular basement membranes ubiquitously express matrix protein low-expression regions: characterization in multiple tissues and remodeling during inflammation. Am J Pathol 2010; 176:482-495.
43 Wang S, Voisin MB, Larbi KY, Dangerfield J, Scheiermann C, Tran M, et al: Venular basement membranes contain specific matrix protein low expression regions that act as exit points for emigrating neutrophils. J Exp Med 2006;203:1519-1532.

44 Proebstl D, Voisin MB, Woodfin A, Whiteford J, D'Acquisto F, Jones GE, et al: Pericytes support neutrophil subendothelial cell crawling and breaching of venular walls in vivo. J Exp Med 2012;209:1219-1234.

45 Feng D, Nagy JA, Pyne K, Dvorak HF, Dvorak AM: Neutrophils emigrate from venules by a transendothelial cell pathway in response to FMLP. J Exp Med 1998;187:903-915.

-46 Sumagin R, Sarelius IH: Intercellular adhesion molecule-1 enrichment near tricellular endothelial junctions is preferentially associated with leukocyte transmigration and signals for reorganization of these junctions to accommodate leukocyte passage. J Immunol 2010;184:5242-5252.

47 Soehnlein O, Lindbom L, Weber C: Mechanisms underlying neutrophil-mediated monocyte recruitment. Blood 2009;114: 4613-4623.

48 Mydel P, Shipley JM, Adair-Kirk TL, Kelley DG, Broekelmann TJ, Mecham RP, et al: Neutrophil elastase cleaves laminin-332 (laminin-5) generating peptides that are chemotactic for neutrophils. J Biol Chem 2008;283: 9513-9522.

49 Rowe RG, Weiss SJ: Breaching the basement membrane: who, when and how? Trends Cell Biol 2008; 18:560-574.

50 Hyun YM, Sumagin R, Sarangi PP, Lomakina E, Overstreet MG, Baker CM, et al: Uropod elongation is a common final step in leukocyte extravasation through inflamed vessels. J Exp Med 2012;209:1349-1362.

- 51 Voisin MB, Woodfin A, Nourshargh S: Monocytes and neutrophils exhibit both distinct and common mechanisms in penetrating the vascular basement membrane in vivo. Arterioscler Thromb Vasc Biol 2009;29: 1193-1199.
2 Friedl P, Weigelin B: Interstitial leukocyte migration and immune function. Nat Immunol 2008;9:960-969.

-53 Stark K, Eckart A, Haidari S, Tirniceriu A, Lorenz $\mathrm{M}$, von Bruhl ML, et al: Capillary and arteriolar pericytes attract innate leukocytes exiting through venules and 'instruct' them with pattern-recognition and motility programs 1. Nat Immunol 2013;14:41-51.

54 Stramm LE, Li W, Aguirre GD, Rockey JH: Glycosaminoglycan synthesis and secretion by bovine retinal capillary pericytes in culture. Exp Eye Res 1987;44:17-28.

55 Kowluru RA, Zhong Q, Kanwar M: Metabolic memory and diabetic retinopathy: role of inflammatory mediators in retinal pericytes. Exp Eye Res 2010;90:617-623.

56 Fabry Z, Sandor M, Gajewski TF, Herlein JA, Waldschmidt MM, Lynch RG, Hart MN: Differential activation of Th1 and Th2 CD4+ cells by murine brain microvessel endothelial cells and smooth muscle/pericytes. J Immunol 1993;151:38-47.

57 Balabanov R, Beaumont T, Dore-Duffy P: Role of central nervous system microvascular pericytes in activation of antigen-primed splenic T-lymphocytes. J Neurosci Res 1999; 55:578-587.

58 Brady HR, Denton MD, Jimenez W, Takata S, Palliser D, Brenner BM: Chemoattractants provoke monocyte adhesion to human mesangial cells and mesangial cell injury. Kidney Int 1992;42:480-487.

59 Dahlman-Ghozlan K, Ortonne JP, Heilborn JD, Stephansson E: Altered tissue expression pattern of cell adhesion molecules, ICAM-1, E-selectin and VCAM-1, in bullous pemphigoid during methotrexate therapy. Exp Dermatol 2004;13:65-69. 